\title{
NUMERICAL RANGES IN LOCALLY M-CONVEX ALGEBRAS. I
}

\author{
THANASSIS CHRYSSAKIS
}

(Received 3 February 1983; revised 13 April 1984)

Communicated by J. F. Price

\begin{abstract}
The bidual $E_{b}^{\prime \prime}$ of a unital infrabarrelled l.m.c. $C^{*}$-algebra $E$, equipped with the bidual topology and the regular Arens product, is always an l.m.c. $C^{*}$-algebra. On the other hand, a unital l.m.c. *-algebra $E$ has the $C^{*}$-property if and only if every self-adjoint element $x$ of $E$ is strongly hermitian ( $x$ has real numerical range), or the sets of normalized states and normalized continuous positive linear forms of $E$ coincide. Finally, every unital complete l.m.c. $C^{*}$-algebra satisfying, locally, the property 'the extreme points are dense in the set of continuous positive linear forms" (antiliminal algebra) has the complexes as its only normal elements.
\end{abstract}

1980 Mathematics subject classification (Amer. Math. Soc.): 46 H 99, 46 K 99.

Keywords and phrases: numerical range, normalized states, l.m.c. *-algebra, l.m.c. $C^{*}$-algebra.

\section{Introduction}

The present paper deals with the study of numerical ranges within the context of l.m.c. *-algebras (respectively l.m.c. $C^{*}$-algebras). It thus provides results which

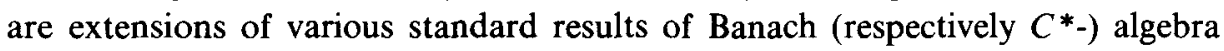
theory. More precisely, one has the following: let $E$ be a unital infrabarrelled l.m.c. $C^{*}$-algebra and $E_{b}^{\prime \prime} \equiv\left(E_{b}^{\prime}\right)_{b}^{\prime}$, the bidual algebra of $E$ equipped with the bidual topology $b$ (topology of uniform convergence on the bounded subsetes of $E_{b}^{\prime}$, the strong dual of $E$ ). Then, if the Arens product on $E_{b}$ is regular, $E_{b}$ (with a natural involution) is a unital l.m.c. $C^{*}$-algebra (Theorem 3.4). This extends the

This paper is partly based on the author's Ph.D. Thesis, the University of Athens.

(C) 1986 Australian Mathematical Society 0263-6115/86\$A2.00+0.00 
analogous result for $C^{*}$-algebras in Bonsall-Duncan (1971), page 110, Theorem 5 . Furthermore, let $E$ be a unital l.m.c. algebra with an involution and $D\left(E, 1_{E}\right)$, $\mathscr{P}(E)$ the sets of normalized states and normalized continuous positive linear forms of $E$, respectively. Then $E$ has the $C^{*}$-property if and only if $D\left(E, 1_{E}\right)$ and $\mathscr{P}(E)$ are the same (Theorem 3.7). The latter contsitutes an extension as well as a strengthening of a theorem by $\mathrm{G}$. Lumer for unital normed *-algebras (Lumer (1961), page 40, Theorem 19). Moreover, $E$ has the $C^{*}$-property if and only if every self-adjoint element of $E$ is strongly hermitian (Theorem 3.7, (1) $\Leftrightarrow(3))$ or even if $E$ satisfies the apparently weaker condition "the set of strongly hermitian self-adjoint elements of $E$ is dense in the set of self-adjoint elements of $E^{\prime \prime}$ (cf. Corollary 3.9). The last equivalences are obtained by applying a different and rather simpler technique than that of B. Sims (1971), and we thereby get an extension to our case of T. W. Palmer's classical characterization of $C^{*}$-algebras (cf., for instance, Sims (1971), Theorem A).

Now let $E$ be a unital complete l.m.c. $C^{*}$-algebra, let $a \in E$, and let $F$ be the closed subalgebra of $E$ generated by $1_{E}, a$ and the elements of $\pi_{E}(a) \equiv$ $\left\{\left(a-\lambda \cdot 1_{E}\right)^{-1} \in E: \lambda \notin \operatorname{sp}_{E}(a)\right\}$, where $\operatorname{sp}_{E}(a)$ is the spectrum of $a$. Then, if $F$ has the property

the set of extreme points of $F$ is dense in the set of continuous positive linear forms of $F$,

the numerical range and the spectrum of $a$ are equal. We thus extend to unital complete 1.m.c. algebras (Theorem 4.7) a criterion of commutativity for Banach algebras due to le Page (Bonsall and Duncan (1971), page 32, Theorem 10 and Srinivasacharyulu (1974), Theorem 1). So every unital complete 1.m.c. $C^{*}$-algebra $E$ which satisfies (I) for each $a \in E$ has as normal elements only the complexes (Corollary 4.8).

Algebras with property (I) are, for instance, the antiliminal algebras (BratteliRobinson (1979), page 346), which are known as "quasi-local" $C$ *-algebras (ibid., pages 121,129$)$ and are particularly used in the axiomatic foundations of quantum mechanics (ibid).

\section{Preliminaries}

The topological algebras considered throughout are complex and Hausdorff with identity elements.

By a locally m-convex (1.m.c.) algebra we mean a topological algebra $E$ whose topology is defined by (an upper directed) family, say $\Gamma \equiv\left\{p_{\alpha}\right\}_{\alpha \in I}$, of submultiplicative semi-norms such that $p\left(1_{E}\right)=1$ (Michael (1952) and Brooks (1967), Theorem 3.2). 
Now let $E$ be a locally convex (1.c.) algebra (an algebra whose underlying topological vector space is locally convex) with continuous multiplication, and let $E^{\prime}, E^{\prime \prime} \equiv\left(E_{b}^{\prime}\right)^{\prime}$ be the dual and bidual space of $E$, respectively, where $b \equiv$ $\beta\left(E^{\prime}, E\right)$ is the dual topology on $E^{\prime}$ (the topology of uniform convergence on the bounded subsets of $E$, cf. Horváth (1966), page 209, and Gulick (1966), page 73). Given $f \in E^{\prime}, x \in E$ and $F \in E^{\prime \prime}$, we define the elements $\langle f, x\rangle$ and $[F, f]$ of $E^{\prime}$ as follows:

$$
\begin{gathered}
\langle f, x\rangle(y):=f(x y), \quad y \in E, \\
{[F, f](y):=F(\langle f, y\rangle), \quad y \in E .}
\end{gathered}
$$

Then the Arens product on $E^{\prime \prime}$ is defined by

$$
(F G)(f)=F([G, f]), \quad f \in E^{\prime},
$$

so that $E^{\prime \prime}$ equipped with this product becomes an algebra, and the canonical map

$$
i: E \rightarrow \hat{E} \subseteq E^{\prime \prime}: x \rightarrow i(x)=\hat{x}
$$

$\left(\hat{x}(f):=f(x), f \in E^{\prime}\right)$ an algebra isomorphism of $E$ onto the subalgebra $\hat{E}$ of $E^{\prime \prime}$ (Gulick (1966), Theorem 3.5). We call this algebra the Arens algebra corresponding to $E$.

On the other hand, the algebra $E^{\prime \prime}$ endowed with the bidual topology (the topology of uniform convergence on the $b \equiv \beta\left(E^{\prime}, E\right)$-bounded subsets of $E^{\prime}$, cf. Gulick (1966), page 74 and Horváth (1966), page 220) becomes a l.c. algebra with a separately continuous multiplication (Gulick (1966), Theorem 3.5), called the locally convex Arens algebra corresponding to $E$. In particular, $E_{b}^{\prime \prime}$ is an 1.m.c. algebra if and only if $E$, equipped with the strong topology $b \equiv \beta^{*}\left(E, E_{b}^{\prime}\right)$ (the topology of uniform convergence on the bounded subsets of $E_{b}^{\prime}$ endowed with the dual topology), is a l.m.c. algebra (Gulick (1966), pages 73, 77).

If a l.m.c. algebra $E$ is, moreover, infrabarrelled (the underlying l.c. space $E$ is infrabarrelled), then the topology $b \equiv \beta^{*}\left(E, E_{b}^{\prime}\right)$ coincides with the initial topology of $E$ (Horvath (1966), page 220, Exercise 5), so that the Arens algebra $E_{b}^{\prime \prime}$ is, in fact, a l.m.c. algebra.

\section{L.m.c. $C^{*}$-algebras}

By an l.m.c. $C^{*}$-algebra we mean an 1.m.c. algebra $E \equiv\left(E,\left\{p_{\alpha}\right\}_{\alpha \in I}\right)$ with an involution * (l.m.c. *-algebra) such that $p_{\alpha}\left(x^{*} x\right)=p_{\alpha}(x)^{2}$ for any $x \in E$, $\alpha \in I$. Now let $E$ be an 1.m.c. algebra and let $\alpha \in I$. Then we define

$$
D_{p_{\alpha}}\left(E, 1_{E}\right) \equiv\left\{f \in\left(U_{p_{\alpha}}(1)\right)^{0}: f\left(1_{E}\right)=1\right\}, \quad \alpha \in I,
$$

where $\left(U_{p_{\alpha}}(1)\right)^{0}$ is the polar of $U_{p_{\alpha}}(1) \equiv\left\{x \in E: p_{\alpha}(x)<1\right\}$. 
The elements of the set

$$
D\left(E, 1_{E}\right) \equiv \bigcup_{\alpha} D_{p_{\alpha}}\left(E, 1_{E}\right)
$$

will be called normalized states of $E$. If $\tilde{E}$ is the completion of $E$, then one has

$$
D\left(E, 1_{E}\right) \equiv D\left(\tilde{E}, 1_{E}\right)
$$

within a bijection.

Definition 3.1. Let $E$ be an l.m.c. algebra. We define the numerical range of an element $\alpha \in E$ to be the set

$$
V_{E}(a)=\hat{a}\left(D\left(E, 1_{E}\right)\right)
$$

with $\hat{a}$ defined as in (2.4). The number

$$
\nu_{E}(a)=\operatorname{Sup}_{\lambda \in V_{E}(a)}|\lambda|
$$

is then called the numerical radius of $a$.

For the case of a normed algebra, see Bonsall-Duncal (1971), Definition 2.1. The above definition is essentially the same as that given by Giles and Koehler (1973), since $f \in\left(U_{p_{\alpha}}(a)\right)^{0}$ if and only if $|f(x)| \leqslant p_{\alpha}(x), x \in E$ (Mallios (1986), page 2, Lemma 1.2).

The following theorem and Corollary 3.3, extend the analogous Banach algebra results (Bonsall-Duncan (1971), Theorem 12.2 and Corollary 12.3, respectively).

THEOREM 3.2. Let $E$ be an infrabarrelled l.m.c. algebra and $E_{b}^{\prime \prime}$ the corresponding l.m.c. Arens algebra (see comments before Section 3). Then, for every $F \in E_{b}^{\prime \prime}$, one has

$$
V_{E_{b}^{\prime \prime}}(F)=\left\{F(f): f \in D\left(E, 1_{E}\right)\right\} .
$$

PRoof. Let $F \in E_{b}^{\prime \prime}$. Since $\hat{E}_{b}$ is dense in $E_{b}^{\prime \prime}$, there is a net $\left(\hat{x}_{i}\right)$ in $\hat{E}_{b} \subseteq E_{b}^{\prime \prime}$ with $\hat{x}_{i} \rightarrow \vec{b} F$. Hence,

$$
\begin{aligned}
V_{E_{b}^{\prime \prime}}(F) & =\left\{T(F): T \in D\left(E_{b}^{\prime \prime}, \hat{1}_{E}\right)\right\}=\left\{T\left(\lim _{i} \hat{x}_{i}\right): T \in D\left(E_{b}^{\prime \prime}, \hat{1}_{E}\right)\right\} \\
& =\left\{\lim _{i} \hat{f}\left(\hat{x}_{i}\right):\left.\hat{f} \equiv T\right|_{\hat{E}_{b}} \in D\left(\hat{E}_{b}, 1_{E}\right)\right\} \quad \text { by }(3.3) \\
& =\left\{\lim _{i} \hat{x}_{i}(f): f \in D\left(E_{b}, 1_{E}\right)\right\} \quad \text { by }(2.4) \\
& =\left\{F(f): f \in D\left(E, 1_{E}\right)\right\} .
\end{aligned}
$$

Corollary 3.3. Let $E, E_{b}^{\prime \prime}$ be as in Theorem 3.2. Then $F \in E_{b}^{\prime \prime}$ is "strongly hermitian" $\left(V_{E_{b}^{\prime \prime}}(F) \subseteq \mathbb{R}\right)$ if and only if $F(f) \in \mathbb{R}$ for every $f \in D\left(E, 1_{E}\right)$. 
Now, a new product $F \cdot G$ may be defined on $E^{\prime \prime}$ by replacing (2.1), (2.2), (2.3) by

$$
\begin{gathered}
\langle f \mid x\rangle(y):=f(y x), \quad y \in E, \\
{[F \mid f](y):=F(\langle f \mid y\rangle), \quad y \in E,} \\
(F \cdot G)(f):=F([G \mid f]), \quad y \in E,
\end{gathered}
$$

respectively. In general $F \cdot G \neq G F$, for $F, G \in E^{\prime \prime}$. In case $F \cdot G=G F$, for $F$, $G \in E^{\prime \prime}$, the Arens product is called a regular product (Bonsall and Duncan (1971), page 107).

Now let $E$ be a 1.c. *-algebra, i.e. $E$ is a 1.c. algebra with involution *. Then, if we define

$$
\begin{aligned}
f^{*}(x) & :=\overline{f\left(x^{*}\right)}, \quad x \in E, \\
F^{*}(f) & =\overline{F\left(f^{*}\right)}, \quad f \in E^{\prime},
\end{aligned}
$$

the map

$$
F \rightarrow F^{*}: E^{\prime \prime} \rightarrow E^{\prime \prime}
$$

is antilinear and satisfies $\left(F^{*}\right)^{*}=F$. However, it is not true in general that $(F G)^{*}=G^{*} F^{*}$ unless the Arens product on $E^{\prime \prime}$ is regular. In this case (3.12) is in fact an involution (Bonsall-Duncan (1971), page 109), and $E^{\prime \prime}$ is then called a *-Arens algebra. So, if $E$ is, moreover, an l.m.c. algebra, and if the respective Arens product is regular, then the corresponding Arens algebra $E^{\prime \prime}$ (with bidual topology) is an 1.m.c. *-Arens algebra.

The next theorem, the proof of which is given after Lemmas 3.5 and 3.6, extends a result of Bonsall and Duncan for the bidual of a $C^{*}$-algebra (Bonsall and Duncan (1971), page 110, Theorem 12.5).

THEOREM 3.4. Let E be an infrabarrelled l.m.c. $C^{*}$-algebra such that the Arens product on $E^{\prime \prime}$ is regular. Then $E_{b}^{\prime \prime}$ (with the Arens involution) is an l.m.c. $C^{*}$-algebra.

Let $E \equiv\left(E,\left\{p_{\alpha}\right\}_{\alpha \in I}\right)$ be a 1.m.c. algebra and let $N_{\alpha}=\operatorname{ker}\left(p_{\alpha}\right), \alpha \in I$. If $E_{\alpha}=E / N_{\alpha}, \alpha \in I$, and if $\|\cdot\|$ is the norm on $E_{\alpha}$ defined by $\left\|x_{\alpha}\right\|_{\alpha}=p_{\alpha}(x)$, where $x_{\alpha}=x+N_{\alpha} \in E_{\alpha}$, then every $E_{\alpha}, \alpha \in I$, is a normed algebra. Now, for $\alpha \leqslant \beta, \alpha, \beta \in I\left(\operatorname{ker}\left(p_{\beta}\right) \subseteq \operatorname{ker}\left(p_{\alpha}\right)\right)$, the surjective homomorphism

$$
f_{\alpha \beta}: E_{\beta} \rightarrow E_{\alpha}: x_{\beta} \rightarrow x_{\alpha}
$$

is well defined (Michael (1952) and Mallios (1986)), and the families $\left(E_{\alpha}, f_{\alpha \beta}\right)$, $\left(\tilde{E}_{\alpha}, \tilde{f}_{\alpha \beta}\right)\left(\tilde{E}_{\alpha}, a \in I\right.$, denotes the completion of $E_{\alpha}$, and $\tilde{f}_{\alpha \beta}, \alpha \leqslant \beta$, the extension of $f_{\alpha \beta}$ to the respective completions) constitute projective systems of normed and Banach algebras, respectively, corresponding to $E$ (Michael (1952)). Moreover, we 
have $E \subseteq \underset{\leftarrow}{\lim } E_{\alpha} \subseteq \underset{\leftarrow}{\lim } \tilde{E}_{\alpha}=\tilde{E}$, where $\tilde{E}$ is the completion of $E$ (Michael (1952) and Mallios (1986)).

Furthermore $E$ is an 1.m.c. $C^{*}$-algebra if and only if every normed algebra ( $E_{\alpha}$, $\left.\|\cdot\|_{\alpha}\right)$ has the $C^{*}$-property $\left(\left\|x_{\alpha}^{*} x_{\alpha}\right\|_{\alpha}=\left\|x_{\alpha}\right\|_{\alpha}^{2}, x_{\alpha} \in E_{\alpha}\right)$.

Now let $D\left(E, 1_{E}\right)$ and $\mathscr{P}(E)$ be, respectively, the normalized states of $E$ (cf. (3.1), (3.2)) and the normalized continuous positive linear forms on $E$ ( $f \in E^{\prime}$ with $f\left(1_{E}\right)=1$ and $\left.f\left(x^{*} x\right) \geqslant 0, x \in E\right)$. Then one has the following.

Lemma 3.5. On every l.m.c. $C^{*}$-algebra $E$, the sets of normalized states and normalized continuous positive linear forms on $E$ coincide, that is

$$
D\left(E, 1_{E}\right)=\mathscr{P}(E) \text {. }
$$

Proof. Let $f \in D_{p_{\alpha}}\left(E, 1_{E}\right) \subseteq D\left(E, 1_{E}\right), \alpha \in I$ (cf. (3.1), (3.2)). Then the function

$$
f_{\alpha}: E_{\alpha} \rightarrow \mathbb{C}: x_{\alpha} \equiv x+N_{\alpha} \rightarrow f_{\alpha}\left(x_{\alpha}\right):=f(x)
$$

is a normalized state of $E_{\alpha}$, and its extension to $\tilde{E}_{\alpha}$ (also denoted by $f_{\alpha}$ ) is a normalized state of the $C^{*}$-algebra $\tilde{E}_{\alpha}, \alpha \in I$. But

$$
D\left(\tilde{E}_{\alpha}, 1_{\alpha}\right)=\mathscr{P}\left(\tilde{E}_{\alpha}\right), \quad \alpha \in I,
$$

(Berberian (1974), Theorem 61.9), so that $f\left(x^{*} x\right) \equiv f_{\alpha}\left(x_{\alpha}^{*} x_{\alpha}\right) \geqslant 0, x \in E$, and hence $f \in \mathscr{P}(E)$.

Conversely, if $f \in \mathscr{P}(E)$, then there is $\alpha \in I$ such that $f_{\alpha} \in \mathscr{P}\left(E_{\alpha}\right)$ (cf. (3.15) and Fragoulopoulou (1981), Theorem 3.1). In particular, the extension of $f_{\alpha}$ to $\tilde{E}_{\alpha}$ is an element of $\mathscr{P}\left(\tilde{E}_{\alpha}\right)$ (ibid.), so that by (3.16) $f_{\alpha} \in D\left(\tilde{E}_{\alpha}, 1_{\alpha}\right)$, and hence $\left\|f_{\alpha}\right\|=1$. Thus $|f(x)|=\left|f_{\alpha}\left(x_{\alpha}\right)\right| \leqslant\left\|x_{\alpha}\right\|_{\alpha} \equiv p_{\alpha}(x), x \in E$, i.e. $f \in D_{p_{\alpha}}\left(E, 1_{E}\right) \subseteq$ $D\left(E, 1_{E}\right)$.

LEMMA 3.6. Let $E$ be an infrabarrelled l.m.c. $C^{*}$-algebra, and assume that the Arens-product on $E_{b}^{\prime \prime}$ is regular (see comments after Corollary 3.3). Then every self-adjoint element of $E_{b}^{\prime \prime}$ is strongly hermitian.

Proof. Let $F \in E_{b}^{\prime \prime}$ be such that $F^{*}=F$. By Corollary 3.3 it is sufficient to prove that $F(f) \in \mathbb{R}$ for every $f \in D\left(E_{b}, 1_{E}\right)$. Let $f \in D\left(E_{b}, 1_{E}\right)$. By hypothesis and by Lemma 3.5 we have that $f \in \mathscr{P}(E)$ (cf. also Horváth (1966), page 220, Exercise 5). Thus, by (3.10), (3.11) and Fragoulopoulou (1981), Lemma 3.3, we get $f=f^{*}$ and $F(f)=F^{*}(f)=\overline{F\left(f^{*}\right)}=\overline{F(f)}$, that is, $F(f) \in \mathbb{R}$ for every $f \in D\left(E_{b}, 1_{E}\right)$.

Proof of Theorem 3.4. For every $x \in E_{b}^{\prime \prime}$ one has

$$
x=y+i z
$$


with $y, z \in S\left(E_{b}^{\prime \prime}\right)$ (self-adjoint elements of $\left.E_{b}^{\prime \prime}\right)$. By Lemma 3.6, $y, z \in H\left(E_{b}^{\prime \prime}\right)$ (strongly hermitian elements of $E_{b}^{\prime \prime}$ ), so that the decomposition (3.17) of $x$ is unique. In fact, if $x=y^{\prime}+i z^{\prime}, y^{\prime}, z^{\prime} \in H\left(E_{b}^{\prime \prime}\right)$ then $y-y^{\prime}, z-z^{\prime} \in H\left(E_{b}^{\prime \prime}\right)$, so that $V_{E_{b}^{\prime \prime}}\left(y-y^{\prime}\right)=(\widehat{y-y})\left(D\left(E_{b}^{\prime \prime}, 1_{E^{\prime \prime}}\right)\right)=\left\{(\overrightarrow{y-y})(f): f \in D\left(E_{b}^{\prime \prime}, 1_{E^{\prime \prime}}\right)\right\}=$ $\left\{f\left(y-y^{\prime}\right): \quad f \in D\left(E_{b}^{\prime \prime}, 1_{E^{\prime \prime}}\right)\right\}=\left\{f\left(i\left(z^{\prime}-z\right)\right): f \in D\left(E_{b}^{\prime \prime}, 1_{E^{\prime \prime}}\right)\right\}=\left\{i \cdot f\left(z^{\prime}-\right.\right.$ $\left.z): f \in D\left(E_{b}^{\prime \prime}, 1_{E^{\prime \prime}}\right)\right\}=i\left\{\left(\overline{z^{\prime}-z}\right)(f): f \in D\left(E_{b}^{\prime \prime}, 1_{E^{\prime \prime}}\right)\right\}=i \cdot V\left(z^{\prime}-z\right) \subseteq \mathbb{R} \cap$ $i \mathbb{R}=\{0\}$. Hence, by Giles and Koehler (1973), Corollary $1, y-y^{\prime}=z-z^{\prime}=0$. Consequently, the Vidav-Palmer involution may be defined on $E_{b}^{\prime \prime}\left(x_{*}=y-i z\right)$, and it actually coincides with the Arens involution. Now the Vidav-Palmer theorem (Giles and Koehler (1973), Theorem 6) guarantees that $E_{b}^{\prime \prime}$ is an 1.m.c. $C^{*}$-algebra.

We shall now give some characterizations of the $C^{*}$-property on an 1.m.c. *-algebra.

THEOREM 3.7. Let E be an l.m.c. *-algebra. Then the following conditions are equivalent.

(1) $E$ is an l.m.c. $C^{*}$-algebra.

(2) $D\left(E, 1_{E}\right)=\mathscr{P}(E)$.

(3) Every self-adjoint element of $E$ is strongly hermitian.

(4) $S(E) \equiv H(E)$

(5) The next two relations hold true

$$
\begin{gathered}
E=H(E) \oplus i \cdot H(E), \\
H(E) \subseteq S(E) .
\end{gathered}
$$

Proof. (1) $\Rightarrow(2)$ : This is Lemma 3.5 .

$(2) \Rightarrow(3)$ : Let $x \in S(E)$. Then by (2) and by Fragoulopoulou (1981), Lemma 3.3, we have $f(x) \in \mathbb{R}$ for every $f \in D\left(E, 1_{E}\right)$, so that $V_{E}(x) \subseteq \mathbb{R}$ by (3.4).

$(3) \Rightarrow(4)$ : let $x \in H(E)$. Then

$$
x=y+i z
$$

where $y, z \in S(E)$, so that, by (3), $y, z \in H(E)$.

On the other hand, $V_{E}(x)=V_{E}(y)+i V_{E}(z)$, and since $x, y, z \in H(E)$, we have $V_{E}(z)=0$, whence (Giles and Koehler (1973), page 34) $z=0$. Therefore $x=y \in S(E)$.

$(4) \Rightarrow(5)$ : by (4) and (3.20) we have that $E=H(E)+i H(E)$. Using the argument in the proof of Theorem 3.4, one gets the uniqueness of (3.20), which proves (3.18).

(5) $\Rightarrow$ (1): The Vidav-Palmer Theorem (Giles and Koehler (1973), Theorem 6) guarantees that $E$ (with the Vidav-Palmer involution) becomes an l.m.c. $C^{*}$-algebra. On the other hand, (3.19) implies that the Vidav-Palmer involution coincides with the given one. 
Scholium 3.8. In the above theorem, condition (3.18) is sufficient to make $E$ (with the Vidav-Palmer involution) into an l.m.c. $C^{*}$-algebra (Giles and Koehler (1973), Theorem 6). But in general the Vidav-Palmer involution does not coincide with the given one, the letter being ensured by (3.19). On the other hand, condition (3.19) is not sufficient by itself to imply (1) of Theorem 3.7, as shown by an example of B. Sims for Banach algebras (Sims (1971), page 198).

COROLLARY 3.9. Let $E$ be an l.m.c. *-algebra with continuous involution. Then $E$ has the $C^{*}$-property if and only if

$$
\overline{S(E) \cap H(E)}=S(E) \text {. }
$$

Proor. Theorem 3.7, (1) $\Rightarrow(4)$, and the fact that $S(E)$ is closed imply (3.21). Conversely, if (3.21) is valid, then the conclusion follows from Theorem 3.7, (3) $\Rightarrow$ (1), and from Giles and Koehler (1973), Lemma 3.

In Theorem 3.7, the equivalence $(1) \Leftrightarrow(2)$ is an extension as well as a strengthening of Lumer (1961), Theorem 19 and Berberian (1974), Theorem 61.9 for normed *-algebras. On the other hand, the equivalence $(1) \Leftrightarrow(3)$ of the same theorem extends to our situation a result of $\mathrm{T}$. W. Palmer for Banach algebras (Sims (1971), Theorem A).

\section{Commutative l.m.c. $C^{*}$-algebras}

Let $E$ be a (non-commutative) algebra and $\left\{a_{1}, a_{2}, \ldots, a_{n}\right\}$ a commutative subset of $E$. Also let

$$
\pi_{E}\left(a_{i}\right)=\left\{\left(a_{i}-\lambda 1_{E}\right)^{-1}: \lambda \notin \mathrm{Sp}_{E}\left(a_{i}\right)\right\}, \quad i=1,2, \ldots, n .
$$

Then the elementds of $\pi_{E}\left(a_{i}\right)$ commute with $a_{1}, a_{2}, \ldots, a_{n}$, as well as with one another, so that the subalgebra $A$ of $E$ generated by $\pi_{E}\left(a_{i}\right), i=1, \ldots, n$, and $1_{E}$, $a_{1}, \ldots, a_{n}$ is commutative.

Proposition 4.1. Let $E$ be an l.m.c. algebra and $\left\{a_{1}, \ldots, a_{2}, \ldots, a_{n}\right\} a$ commutative subset of $E$. if $\mathfrak{A}$ is a commutative closed subalgebra (of $E$ ) containing the previously constructed (commutative) subalgebra $A$, then

$$
\operatorname{Sp}_{\mathfrak{X}}\left(a_{i}\right)=\operatorname{Sp}_{E}\left(a_{i}\right), \quad i=1,2, \ldots, n \text {. }
$$

Proof. Note that $\operatorname{Sp}_{E}\left(a_{i}\right) \subseteq \operatorname{Sp}_{\mathfrak{A}}\left(a_{i}\right)$. On the other hand, if $\lambda \in \operatorname{Sp}_{\mathfrak{A}}\left(a_{i}\right)$ and $\lambda \notin \operatorname{Sp}_{E}\left(a_{i}\right)$, then $\left(a_{i}-\lambda 1_{E} 1_{E}\right)^{-1}$ exists in $E$, so that, by hypothesis for $\mathscr{A}$, we have $\left(a_{i}-\lambda i_{E}\right)^{-1} \in \mathfrak{U}$, i.e. $\lambda \notin \operatorname{Sp}_{\mathfrak{X}}\left(a_{i}\right)$, which is a contradiction. 
Proposition 4.2. Let $E$ be an l.m.c. algebra and $F \equiv\left(F,\left\{p_{\alpha \mid F}\right\}\right)$ an l.m.c. subalgebra of $E$ which contains the unit of $E$. Then, for each $b \in F$, we have

$$
V_{E}(b)=V_{F}(b) \text {. }
$$

Proof. For every $f \in D\left(E, 1_{E}\right)$, we have $\left.g \equiv f\right|_{F} \in D\left(F, 1_{E}\right)$. Conversely, for every $g \in D\left(F, 1_{E}\right)$, the Hahn-Banach Theorem implies the existence of an $f \in D\left(E, 1_{E}\right)$ with $g=\left.f\right|_{F} \in D\left(E, 1_{E}\right)$, so that the result follows from (3.4).

In quantum mechanics one meets $C^{*}$-algebras satisfying the following condition (Bratteli and Robinson (1979), pages 345-346).

The set $\mathscr{B}(E)$ of extreme points of $\mathscr{P}(E)$ is a dense subset of $\mathscr{P}(E)$.

In this concern, one has

Proposition 4.3. In every complete commutative l.m.c. $C^{*}$-algebra E, the set $\mathfrak{B}(E)$ is weakly closed.

Proof. If $\mathfrak{M}(E)$ is the topological spectrum of $E$ (the set of nonzero continuous characters of $E$ ), then (Brooks (1967), Theorem 4.5)

$$
\mathscr{P}(E) \cap \mathfrak{M}(E)=\mathfrak{B}(E) .
$$

On the other hand, for each $a \in E$,

$$
\operatorname{Sp}_{E}(a)=\hat{a}(\mathfrak{M}(E))=\{f(a): f \in \mathfrak{M}(E)\}
$$

(Mallios (1986), page 104, Corollary 6.4). Particularly, if $a=x^{*} x, x \in E$, one gets (Inoue (1971), Proposition 2.1) $\mathrm{Sp}_{E}(a) \geqslant 0$, so that $\mathfrak{M}(E) \subseteq \mathscr{P}(E)$, and hence, by (4.2),

$$
\mathfrak{R}(E)=\mathfrak{B}(E) .
$$

But $\mathfrak{M}(E)$ is closed in $E_{s}^{\prime}$ (Mallios (1986), page 142, Remark 1.1), so $\mathfrak{B}(E) \subseteq E_{s}^{\prime}$ is closed.

COROllaRY 4.4. Let $E$ be a complete commutative l.m.c. $C^{*}$-algebra with property (4.1). Then, for each $a \in E$, one has

$$
\operatorname{Sp}_{E}(a)=V_{E}(a)
$$

In particular, one has, in effect, $E \cong \mathbb{C}$ (topological algebraic isomorphism).

Proof. By hypothesis we have

$$
\mathfrak{B}(E)=\mathscr{P}(E)=\mathfrak{M}(E)=D\left(E, 1_{E}\right)
$$


(see also Theorem 3.7 and the proof of Proposition 4.3). Furthermore, $E=$ $\mathscr{C}(\mathfrak{M}(E))$ (Gel'fand-Naimark), so that we need prove only that $E$ is, in fact, a division algebra: that is, $\hat{x}(f) \neq 0, f \in \mathfrak{M}(E)$, or, equivalently, $0 \notin \operatorname{Sp}_{E}(x)$, $x \in E$. For if $f(x)=0$ for some $x \in E$ and some $f \in \mathfrak{M}(E)=\mathscr{P}(E)$, let $g \in \mathscr{P}(E)$. Then one concludes from (4.5) that $\lambda_{1} f=\lambda_{2} g=h \in \mathscr{P}(E)=\mathfrak{B}(E)$ $=\mathfrak{M}(E)$ (take, e.g., $h=f+g$ ). Thus $g(x)=0$, that is, $f(x)=0$ for every $f \in \mathscr{P}(E)=D\left(E, 1_{E}\right)$, and so $x=0$.

THEOREM 4.5. Let $E$ be a complete l.m.c. $C^{*}$-algebra and a a normal element of $E\left(a a^{*}=a^{*} a\right)$. Moreover, let $F$ be the closed subalgebra of $E$ generated by $1_{E}, a$, $a^{*}$ and by the elements of $\pi_{E}(a), \pi_{E}\left(a^{*}\right)$. Assume that $F$ satisfies (4.1). Then

$$
\operatorname{Sp}_{E}(a)=V_{E}(a)
$$

Proof. $F$ is a complete l.m.c. $C^{*}$-subalgebra of $E$. Moreover, $F$ is commutative (see comments before Proposition 4.1), so that the conclusion now follows from Corollary 4.4 and from Propositions 4.1, 4.2.

For a discussion of Theorem 4.5 in the case of Banach algebras and complete 1.m.c. algebras, see Bonsall and Duncan (1971), Theorem 6 and Giles and Koehler (1973), Theorem 1, respectively.

The closed subalgebra $F$ of the above theorem will be called the basic subalgebra of $E$ at the normal element $a$. If $F$ satisfies (4.1), we shall say that $E$ satisfies (4.1) locally at the (normal) element $a \in E$. We abbreviate terminology by saying that $E$ satisfies (4.1) locally if this is the case for every normal element of $E$.

In this respect, one already has from Theorem 3.7 the next result.

COROLlaRy 4.6. Let E be a complete l.m.c. $C^{*}$-algebra. Then

$$
S(E)=H(E)=H_{1}(E),
$$

where $H_{1}(E)$ denotes the hermitian elements of $E$ (self-adjoint elements with real spectra).

In the sequel we shall give a criterion of commutativity for l.m.c. algebras (cf. Theorem 4.7), and we shall next prove that the complete l.m.c. $C^{*}$-algebras which satisfy (4.1) have the complexes as the only normal elements (cf. Corollary 4.8).

Let $E$ be a complete l.m.c. algebra and let $a \in E$. Then the exponential function is defined as follows:

$$
\exp (a):=1+\sum_{n=1}^{\infty} \frac{1}{n !} a^{n} .
$$


The existence of the "infinite sum" in (4.8) is a consequence of the fact that, the partial sums $x_{n} \equiv \sum_{1}^{n}(1 / k !) \cdot a^{k}, n=1,2, \ldots$, constitute a Cauchy sequence, which is true by hypothesis.

Now, for any commuting elements $a, b \in E$, one gets

$$
\begin{gathered}
\exp (a+b)=\exp (a) \cdot \exp (b), \\
\exp (a) \cdot \exp (-a)=1 .
\end{gathered}
$$

The proof of (4.9), (4.10) is analogous to that of Bonsall and Duncan (1971), page 26, Theorem 2 for Banach algebras. A consequence of (4.10) is that $\exp (a) \in G(E)$ (the invertible elements of $E$ ). On the other hand, one has that the $E$-valued map $\lambda \rightarrow \exp (\lambda a), \lambda \in \mathbf{C}$, is holomorphic.

THEOREM 4.7. Let $E \equiv\left(E,\left\{p_{\alpha}\right\}_{\alpha \in I}\right)$ be a complete l.m.c. algebra, such that

$$
\rho_{E}(a) \geqslant k \cdot \nu_{E}(a)
$$

for some $k>0$, for all $a \in E$. Then $E$ is commutative.

Proof. Let $a, b \in E$ and define

$$
\phi: \mathbf{C} \rightarrow E: \lambda \rightarrow \phi(\lambda):=\exp (-\lambda a) \cdot b \cdot \exp (\lambda a),
$$

which is a continuous function. Then, if $f \in\left(U_{p_{a}}(1)\right)^{0}, \alpha \in I$, we have $|(f \circ \phi)(\lambda)|$ $\leqslant p_{\alpha}(\phi(\lambda)) \leqslant e \cdot \nu_{E}(\phi(\lambda)), \lambda \in \mathbb{C}$, where $e \simeq 2.73$ (see comments after Definition 3.1 and Giles and Koehler (1973), Lemma 1).

On the other hand, $\operatorname{Sp}_{E}(\phi(\lambda))=\operatorname{Sp}_{E}(b)$, since $\exp (\lambda a) \cdot\left(\phi(\lambda)-\mu \cdot 1_{E}\right)=(b$ $\left.-\mu \cdot 1_{E}\right) \cdot \exp (\lambda a)$, so that $\phi(\lambda)-\mu \cdot 1_{E} \in G(E)$ if and only if $b-\mu \cdot 1_{E} \in$ $G(E)$. Thus,

$$
|(f \circ \phi)(\lambda)| \leqslant(e / k) \cdot \rho_{E}(b) \equiv \lambda \in \mathbb{C},
$$

and hence the function $f \circ \phi$ is bounded. Moreover, $f \circ \phi$ is holomorphic since $\phi$ is, and consequently $f \circ \phi$ is constant by Liouville's theorem. Therefore

$$
f\left(\phi\left(\lambda_{1}\right)\right)=f\left(\phi\left(\lambda_{2}\right)\right), \quad \lambda_{1}, \lambda_{2} \in \mathbb{C},
$$

for all $f \in U_{\alpha \in I}\left(U_{p_{\alpha}}(1)\right)^{0} \subseteq E^{\prime}$. Hence, $g\left(\phi\left(\lambda_{1}\right)-\phi\left(\lambda_{2}\right)\right)=0, \lambda_{1}, \lambda_{2} \in \mathbb{C}$, for all $g \in E^{\prime}$, so that (Hahn-Banach Theorem, Treves (1967), page 187, Corollary 2) $\phi\left(\lambda_{1}\right)=\phi\left(\lambda_{2}\right), \lambda_{1}, \lambda_{2} \in \mathbb{C}$. Thus $b=\phi(0)=\phi(1)=\exp (-a) \cdot b \cdot \exp (a)$, that is, $\exp (a) \cdot b=b \cdot \exp (a)$ for all $a, b \in E$, and consequently $\exp (\lambda a) \cdot b=b$. $\exp (\lambda a), \lambda \in \mathbf{C}$. This in turn implies that

$$
\frac{\exp (\lambda a)-1}{\lambda} \cdot b=b \cdot \frac{\exp (\lambda a)-1}{\lambda}, \quad \lambda \in \mathbb{C},
$$

so that by taking limits we obtain $a \cdot b=b \cdot a, a, b \in E$.

Corollary 4.8. Let E be a complete l.m.c. $C^{*}$-algebra satisfying (4.1) locally. Then the only normal elements of $E$ are the complexes. 
Proof. If $x$ is a normal element of $E$, consider the closed (commutative) subalgebra of $E$ generated by $\left\{1_{E}, x, x^{*}\right\}$ together with the elements of the sets $\pi_{E}(x)$ and $\pi_{E}\left(x^{*}\right)$ (cf. Theorem 4.5). If $F$ denotes the latter algebra, then $F$ satisfies the conditions of Corollary 4.4 , and hence $F \cong \mathbb{C}$.

Now let $E$ be an l.m.c. algebra. Then by (3.5) one has

$$
\begin{gathered}
\nu_{E}(x+y) \leqslant \nu_{E}(x)+\nu_{E}(y), \quad x, y \in E, \\
\nu_{E}(\lambda x)=|\lambda| \cdot \nu_{E}(x), \quad x \in E, \lambda \in \mathbb{C} .
\end{gathered}
$$

Thus, if $\nu_{E}(x)=0$, then $V_{E}(x)=0$, and so $x=0$ (Giles and Koehler (1973), Corollary 1$)$, that is, $\nu_{E}$ is a linear norm.

In this regard, we have the next result.

THEOREM 4.9. Let $E$ be a Fréchet l.m.c. algebra. If the numerical radius $\nu_{E}$ is a complete linear norm (the algebra $\left(E, \nu_{E}\right)$ is a Banach space) then $E$ is a Banachable algebra, i.e. its topology may be defined by a complete algebraic (submultiplicative) norm.

Proof. We have that $\nu_{E}(x) \geqslant(1 / e) \cdot p_{\alpha}(x), x \in E, \alpha \in I$, where $e \simeq 2.73$ (Giles and Koehler (1973), page 84, Corollary 1), so that the identity map

$$
\operatorname{id}_{E}:\left(E, \nu_{E}\right) \rightarrow\left(E,\left\{p_{\alpha}\right\}_{\alpha \in I}\right)
$$

is continuous. Now the open-mapping theorem guarantees that the map $\mathrm{id}_{E}$ is a topological isomorphism, and since $\left(E, \nu_{E}\right)$ is a Banach space, $E$ has a continuous multiplication, so that (Mallios (1986), page 34, Theorem 4.1) there is a new norm, equivalent to $\nu_{E}$, with respect to which $E$ is a Banach algebra.

Now if $E$ is a complete l.m.c. algebra, and if both the spectral and numerical radii $\rho_{E}$ and $\nu_{E}$ are Banach algebra norms for $E$, then $E$ is commutative. Thus (Wenjen (1968), Theorem 1 ), if $E$ is, moreover, a weakly (l.m.c.) $C^{*}$-algebra $\left(p_{\alpha}\left(x^{*} x\right) \geqslant M \cdot p_{\alpha}\left(x^{*}\right) \cdot p_{\alpha}(x), x \in E, \alpha \in I, M>0\right)$ then $E$ is topologically isomorphic with the algebra $\mathscr{C}(T)$ (continuous complex-valued functions on a completely regular space $T$ ) equipped with a topology $\tau \leqslant c$ (topology of compact convergence).

In fact, by Giles and Koehler (1973), Theorem $1, \rho_{E}(x) \leqslant \nu_{E}(x), x \in E$, so that the identity map

$$
\operatorname{id}_{E}:\left(E, \nu_{E}\right) \rightarrow\left(E, \rho_{E}\right)
$$

is continuous, and so (open-mapping theorem) a topological isomorphism; hence, there is $k>0$ with $\rho_{E}(x) \geqslant k \cdot \nu_{E}(x), x \in E$. Thus, by Theorem 4.7, $E$ is commutative. 
The above comments provide an extension as well as an improvement of previous results in Srinivasacharyulu (1974).

\section{Acknowledgement}

The author wishes to express his gratitude to Professor A. Mallios for his helpful guidance, as well as for several stimulating discussions during the preparation of this paper. Suggestions of the referee are also thankfully acknowledged.

\section{References}

S. Berberian (1974), Lectures in functional analysis and operator theory (Springer-Verlag, New York).

F. Bonsall and J. Duncan (1971), Numerical ranges of operators on normed spaces and of elements of normed algebras (London Math. Soc. Lecture Notes, Ser. 2, Cambridge University Press).

O. Bratteli and D. Robinson (1979), Operator algebras and quantum statistical mechanics I (Springer-Verlag, Berlin).

R. M. Brooks (1967), 'On locally $m$-convex *-algebras', Pacific J. Math. 23, 5-23.

M. Fragoulopoulou (1983), 'Spaces of representations and enveloping l.m.c. *-algebras', Pacific J. Math. 95, 61-73.

J. Giles and D. Koehler (1973), 'On numerical ranges of elements of locally $m$-convex algebras', Pacific J. Math. 49, 79-81.

S. L. Gulick (1966), 'The bidual of a locally multiplicatively-convex algebra', Pacific J. Math. 17, $71-76$.

J. Horváth (1966), Topological vector spaces and distributions (Addison-Wesley, Reading, Mass.).

A. Inoue (1971), 'Locally C*-algebras', Mem Fac. of Sci. Kyushu Univ. 25, 197-235.

G. Lummer (1961), 'Semi-inner product spaces', Trans. Amer. Math. Soc. 100, 41-43.

A. Mallios (1986), Topological algebras: selected topics (North-Holland, Amsterdam).

E. Michael (1952), Locally multiplicatively convex topological algebras, (Mem. Amer. Math. Soc., No. 11).

B. Sims (1971), 'A characterization of Banach *-algebras by numerical range', Bull. Austral. Math. Soc. 4, 193-200.

K. Srinivasacharyulu (1974), 'Remarks on Banach algebras', Bull. Soc. Sci. de Liège, 43, 523-525.

F. Treves (1967), Topological vector spaces, distributions and kernels (Academic Press, New York).

Ch. Wenjen (1968), 'A remark on a problem of M. A. Naimark', Proc. Japan Acad. 44, 651-655.

B. Yood (1960), 'Faithful *-representations of normed algebras', Pacific J. Math. 10, 346-363.

Mathematical Institute

University of Athens

57, Solonos Street

Athens 10679

Greece 